$\begin{array}{cl}\begin{array}{c}\text { Revue } \\ \text { de /histoire }\end{array} & \text { Revue de l'histoire des religions } \\ \text { des religions } & \begin{array}{l}3 \mid 2015 \\ \text { Varia }\end{array}\end{array}$

\title{
L'athéisme et le doute au Moyen Âge : un problème controversé
}

Atheism and Doubt in the Middle Ages: A New Approach to a Difficult Subject

Dorothea Weltecke

\section{OpenEdition}

1 Journals

Édition électronique

URL : http://journals.openedition.org/rhr/8409

DOI : $10.4000 /$ rhr.8409

ISSN : 2105-2573

Éditeur

Armand Colin

Édition imprimée

Date de publication : 1 septembre 2015

Pagination : 339-361

ISBN : 978-2-200-92993-0

ISSN : 0035-1423

Référence électronique

Dorothea Weltecke, "L'athéisme et le doute au Moyen Âge : un problème controversé », Revue de I'histoire des religions [En ligne], 3 | 2015, mis en ligne le 01 septembre 2018, consulté le 30 avril 2019. URL : http://journals.openedition.org/rhr/8409 ; DOI : 10.4000/rhr.8409 


\section{L'athéisme et le doute au Moyen Âge: un problème controversé}

L'article esquisse la forme et la diffusion médiévales du phénomène que l'on appelle aujourd'hui "l'athéisme», autrement dit ne pas croire en l'existence d'un dieu. L'auteur étudie des textes pour isoler des expressions et des conceptions de ce phénomène. La traduction du terme «non-croyance/infidelitas» par «athéisme» est critiquée pour son ambiguïté. En outre, plus surprenant, l'étude des sources démontre que la non-croyance n'était ni interdite, ni poursuivie par les autorités ecclésiastiques et séculières. Le phénomène n'était guère pris au sérieux et une discussion rationnelle sur le sujet n'existait quasiment pas. La littérature spirituelle proposait au clergé de réagir à des cas de noncroyance par des exhortations.

\section{Atheism and Doubt in the Middle Ages: A New Approach to a Difficult Subject}

The article outlines the form and distribution of the phenomenon in the Middle Ages known today as 'atheism', i.e. not to believe in the existence of a god. Texts are analysed to isolate terms and concepts for this phenomenon. The translation of the term 'unbelief/infidelitas' with 'atheism' is criticised as misleading as it denoted only non-christian, disloyal or impious individuals, not 'atheists'. The study of legal, inquisitorial and spiritual sources also yields that ecclesiastical and secular powers were not interested to persecute non-believers. There was hardly any rational discussion about it. On the other hand, spiritual care addressed situations of doubt, non-belief and protest by means of exhortation and admonition. 
Dès le début de l'époque moderne, tant les premiers athées euxmêmes que leurs adversaires ont élaboré des théories divergentes quant à l'histoire de l'athéisme. Deux thèses opposées dominent ce champ. En premier lieu, certains soutiennent l'idée qu'au Moyen Âge aussi les athées étaient persécutés par l'usage de la force. En effet, le premier ouvrage athée de l'époque moderne, le Theophrastus redivivus rédigé vers 1650 , narre l'histoire de l'athéisme sous la forme d'une histoire de persécutions. Il est intéressant de constater que cette narration concorde avec celle d'un fervent partisan religieux, François Garasse. Ce jésuite, bien que pour des raisons différentes, faisait aussi état dans ses histoires de la terrible fin qui a toujours foudroyé les impies. Cette théorie semble évidente de prime abord: les tribunaux de l'Inquisition avaient eu pour mission le dépistage de certains mouvements religieux déviants, qui devaient être soumis à la justice séculière ou ecclésiastique ${ }^{1}$.

Cependant, la thèse de la persécution des athées au Moyen Âge a toujours présenté un inconvénient majeur. Il est vrai que les savants médiévaux n'ont pas toujours exposé des points de vue cosmologiques et théologiques orthodoxes, loin de là. Les discussions étaient vives et certains savants sont entrés en conflit avec les autorités ecclésiastiques ou séculières. Mais il n'existe aucune preuve non équivoque démontrant qu'un savant médiéval a explicitement défendu l'idée que Dieu n'existe pas. Winfried Schröder et Martin Mulsow ont démontré qu'aucune théorie athée ne peut être documentée avant le XVII ${ }^{\mathrm{e}}$ siècle ${ }^{2}$. L'absence de preuves documentaires est souvent expliquée par la peur des persécutions. Cet argument ne semble pourtant pas convaincant aux yeux de nombreux savants modernes, compte tenu des autres déclarations

1. Cette contribution résume les résultats de l'étude de Dorothea Weltecke "Der Narr spricht: Es ist kein Gott». Studien zu Atheismus, Unglauben und Glaubenszweifel vom 12. Jahrhundert bis zur Neuzeit, Francfort, Campus, 2010. Voir cette étude pour une documentation détaillée de la littérature et des sources.

2. Winfried Schröder, Ursprünge des Atheismus. Untersuchungen zur Metaphysik und Religionskritik des 17. und 18. Jahrhunderts, Stuttgart-Bad Cannstatt, Frommann-Holzboog («Quaestiones», 11), 1998. Martin Mulsow, Moderne aus dem Untergrund. Radikale Frühaufklärung in Deutschland 1680-1720, Hambourg, Meiner, 2002. 
radicales effectivement attestées par écrit dans les procès-verbaux de l'Inquisition.

La recherche actuelle ne tente plus de découvrir des idées philosophiques athées avant 1500. Il paraît en effet inutile ou peu prometteur de rechercher de telles conceptions avant cette date. Cet état de fait semble au contraire étayer la seconde thèse dominante, contre laquelle certains historiens de la philosophie n'ont cessé d'argumenter: dans un monde tout entier pénétré par l'ordre religieux et empreint de piété - selon les affirmations de Jakob Burckhardt ou Lucien Febvre -, il n'y avait aucune place pour une pensée niant l'existence de Dieu. Cette seconde théorie s'inscrit également dans une tradition qui remonte au début de l'époque moderne. Cent cinquante ans après l'apparition des premiers ouvrages athées, au cours de l'année 1799, au moment de la soi-disant querelle de l'athéisme autour de Fichte, les savants se demandaient encore s'il n'y avait vraiment eu aucun penseur médiéval qui n'eût pas cru en Dieu. Le moment où cette thèse est apparue comme une évidence semble donc plutôt récent.

Or le terme et le concept d' «athéisme» ne sont apparus qu'au $\mathrm{XVI}^{\mathrm{e}}$ siècle. Au début, le terme était un mot étranger qui traduisait le latin impietas. Ce dernier terme avait une signification beaucoup plus large que le terme «athéisme», qui signifie le rejet de l'existence d'un dieu. Le nouveau mot était imprécis. Grâce à Lucien Febvre et Paul Oskar Kristeller, nous prenons beaucoup d'accusations d'athéisme des $\mathrm{XVI}^{\mathrm{e}}$ et $\mathrm{XVII}^{\mathrm{e}}$ siècles pour ce qu'elles sont: des bombes lâchées tous azimuts à seule visée polémique. Le mot «athéisme» pouvait signifier tout et n'importe quoi, mais avant tout un défaut d'orthodoxie ou de piété. Au moyen de cette bombe polémique, les dogmaticiens et les hommes politiques religieux s'attaquaient à leurs adversaires. Or, puisqu'il a fallu des siècles pour définir et préciser la notion d' «athéisme», nous avons de bonnes raisons pour ne pas vouloir nous servir de ce mot. Cette observation ne répond pourtant pas à la question de savoir ce qu'il en était de la non-croyance en Dieu.

En premier lieu, il ne nous semble pas raisonnable de former un concept historiographique en le projetant à partir d'une notion conçue à l'époque moderne. Si la préexistence d'une notion dans le monde historique objet de l'enquête était une condition normative de la recherche historique, celle-ci serait la plupart du temps 
impossible. Ceci est vrai par exemple pour l'histoire comparée des religions. Comme chacun le sait, le terme «religion» est lui aussi un produit de l'époque moderne; il est absent de la plupart des cultures du passé, comme l'est le mot «athéisme».

Cependant, par-delà la question du mot utilisé, cet article traitera plutôt la question de savoir s'il y avait au Moyen Âge des gens qui ne croyaient pas en l'existence d'un dieu. À cet effet, deux présupposés doivent être d'emblée acceptés. En premier lieu, la représentation d'une unité de la foi chrétienne est un mythe moderne. Au $\mathrm{xx}^{\mathrm{e}}$ siècle, l'étude des hérésies et de la piété a suffisamment montré qu'au Moyen Âge beaucoup de gens ne croyaient pas aux principaux dogmes de l'Église romaine. Aucun dogme ne fut incontesté, que ce soit La Trinité, l'incarnation ou la transsubstantiation. De plus, l'Église comme institution a subi des critiques radicales; par intermittence, des protestations violentes se sont même élevées contre des prêtres. Mais celui qui rossait un prêtre et n'allait pas à l'église le dimanche n'était pas forcément un homme qui ne croyait pas en Dieu - au contraire.

En second lieu, l'idée d'une piété naïve et naturelle au Moyen Âge n'est, elle aussi, qu'un mythe moderne. Dans les sources textuelles, on rencontre beaucoup de preuves de négligence envers l'Église, de manque d'intérêt ou même d'ignorance totale concernant la religion. Les dominicains et les franciscains prononçaient leurs premiers sermons sur les places de marché, puisque c'est là que se trouvait leur public.

Pourtant, ces deux présupposés ne permettent pas d'inférer l'existence d'un athéisme médiéval. En effet, des recherches sociologiques récentes montrent que le fait de ne pas aller à l'église n'est pas une preuve d'athéisme. Cette réserve doit également être appliquée au Moyen Âge; des témoignages d'attitudes négatives ne sauraient être suffisants.

Comme seules preuves acceptables, nous ne pouvons prendre en compte que les déclarations concrètes de doutes ou de non-croyance. À cet effet, nous examinerons un certain nombre de sources. Premièrement, nous aborderons la question des mots et des notions en prenant comme point de départ un terme utilisé de plus en plus couramment par la recherche pour décrire l'athéisme médiéval: le terme «incroyance». Deuxièmement, nous nous demanderons comment les médiévaux exprimaient concrètement le sentiment 
qu'il n'y a pas de dieu, avant d'accéder au doute religieux. À propos de l'incroyance et du doute religieux, il est également nécessaire de reconstruire les normes qui ont servi de critères pour la persécution des athées. Finalement, nous décrirons les concepts médiévaux qui expriment littéralement la non-croyance et le doute religieux, et qui peuvent donc être admis comme preuves concrètes de non-croyance.

\section{L'INCROYANCE}

Le terme «incroyance»/infidelitas qui avait différentes significations au Moyen Âge, a joué un rôle important dans le discours scientifique récent ${ }^{3}$. En tant que terme juridique du droit médiéval, il désignait les non-chrétiens, surtout les musulmans, mais aussi les juifs. Une autre signification de ce mot est «qui se joint à un groupe d'hérétiques », «qui est un homme infidèle» - le français a conservé le sens du mot latin - et «qui a accompli une trahison envers le roi ou envers un seigneur». Il peut enfin vouloir dire «qui ne prend pas soin de sa piété ». Des hommes ainsi désignés refusaient d'honorer une relique ou un saint, ou même s'en moquaient. L'hagiographie en abonde. On narrait l'histoire d'horribles punitions divines pour montrer à quel point la fin des moqueurs serait terrible. Mais jamais on ne signifiait avec infidelis/infidelitas, le rejet absolu de l'existence de dieu expressis verbis. Contrairement à ce que l'on pourrait penser, ce sens ne semble pas avoir été intégré au concept d'infidelitas. Quoi qu'il en soit, il importe de signaler que hors de la sphère du droit, le mot infidelitas n'a pas été un concept très répandu. Par exemple, on ne le rencontre pas dans le canon des sept péchés capitaux.

En outre, on place aujourd'hui la non-croyance aux antipodes de la foi. Tel n'était pas le cas au Moyen Âge. Thomas d'Aquin a précisé que le contraire de la fides/foi n'est pas une infidelitas générique - comme on pourrait s'y attendre; il arguait qu'il y a plusieurs formes et natures d'infidelitas, conformément à celles que nous avons dénombrées ci-dessus ${ }^{4}$. Ainsi, au terme «foi» ne

3. Voir Weltecke, «Der Narr spricht: Es ist kein Gott», p. 257-295 avec une documentation détaillée des sources et de l'état de la recherche.

4. Thomas d'Aquin, Summa Theologiae, I-III (« Sancti Thomae Aquinatis Opera Omnia», 4-12) Rome, Befani, 1888-1906, t. II, II-II qu. 10; voir Weltecke, «Der Narr spricht: Es ist kein Gott», p. 277-280. 
s'oppose pas le terme infidelitas, mais l'on rencontre régulièrement le terme idolatria. L'infidelitas n'est que très rarement symbolisée ou figurée. Un exemple du genre est la célèbre fresque de Giotto qui représentait l'infidelitas comme le contraire de la fides. Cependant, un examen plus attentif de la fresque révèle que cette représentation de l'infidelitas n'est pas un athée. Sur cette fresque de l'église de l'Arena de Padoue, la figure de l'infidelitas tient une petite idole dans sa main, à laquelle elle est attachée par une laisse qui la ceint autour du cou. Elle porte un casque ressemblant aux heaumes antiques. Cette figure qui fait face à la foi est en réalité l'idolâtrie captive de la croyance en une illusion créée par elle-même.

Dans la littérature spirituelle, nous constatons que le mot infidelitas ne désignait pas les infidèles (juifs, musulmans ou païens), mais signifiait un manque de piété/une impietas, défaut qui n'était pas communément jugé comme un manquement très grave. L'infidelitas banale n'excluait pas l'homme de la communauté. Manquer de piété faisait partie de la vie humaine et quotidienne. $\mathrm{Du} \mathrm{XIII}^{\mathrm{e}}$ au XV $\mathrm{XV}^{\mathrm{e}}$ siècle, les réformateurs de l'Église étaient d'avis que le manque de piété pouvait être confessé et pardonné.

Au vu de la diversité des significations du mot infidelitas, on doit constater que le terme «incroyance» ne constitue pas un équivalent adéquat et ne peut donc pas aider à démêler l'écheveau de l'étude de la non-croyance. Le mot «incroyance» suggère un phénomène qui n'est pas signifié par le mot infidelitas. En outre, cet outil d'analyse utilisé dans des recherches récentes a déjà donné lieu à des anachronismes. En effet, depuis une vingtaine d'années, le mot infidelitas a été fallacieusement traduit par la notion moderne d'«athéisme» dans les sources latines.

\section{L’INSENSÉ SE dIT : IL N’Y A PAS DE DIEU}

Au Moyen Âge, il n’y a pas de terme spécifique pour signifier la non-croyance en dieu. Celui qui voulait exprimer l'idée que quelqu'un ne croit pas en l'existence d'un dieu devait prononcer une phrase entière, il devait dire qu' un tel ne croit pas qu'il existe un dieu. Et même cette phrase n'était pas sans équivoque. Elle dérive de la traduction latine de la Bible, où dans les psaumes 14 et 53 il est fait mention d'un insipiens, d'une personne insensée, qui dit non 
est deus. De toute évidence, la question qui demande si quelqu'un ne croit pas en dieu, n'est pas traitée dans ce psaume 14 (1-4):

L'insensé dit en son cœur: Il n'y a point de Dieu! Ils se sont corrompus, ils ont commis des actions abominables; Il n'en est aucun qui fasse le bien. L'Éternel, du haut des cieux, regarde les fils de l'homme, Pour voir s'il y a quelqu' un qui soit intelligent, Qui cherche Dieu. Tous sont égarés, tous sont pervertis; Il n'en est aucun qui fasse le bien, Pas même un seul. Tous ceux qui commettent l'iniquité ont-ils perdu le sens? Ils dévorent mon peuple, ils le prennent pour nourriture; Ils n'invoquent point l'Éternel.

De fait, le problème de la non-croyance au sens ontologique ne fut jamais l'objet de l'exégèse juive ${ }^{5}$. Il est ici fait mention de quelqu'un pour qui la question de l'existence de Dieu et de ses commandements n'a pas d'importance. Cet homme ne recherche tout simplement pas Dieu au sens biblique et il est inique à l'égard des hommes démunis et pauvres.

De cette lecture juive dérive la première des deux interprétations majeures attestées dès le commencement de l'exégèse chrétienne dans l'Antiquité tardive. L'insensé fut par exemple représenté dans le psaltérion d'Utrecht produit au Haut Moyen Âge ${ }^{6}$. Il est dépeint comme un tyran qui tolère la mise à mort de gens désarmés, qui ne prend pas les veuves et les orphelins à sa charge et qui laisse libre cours au mal. Au premier plan, divers actes de violence sont représentés, par exemple des rapts. Du fait de cette interprétation qui vaut jusqu'au Moyen Âge tardif, on pouvait critiquer un dirigeant en lui attribuant la proposition «il n'y a pas de Dieu». Dans ce contexte, il n'importait pourtant pas de savoir si un tel dirigeant avait une opinion sur la question de l'existence de dieu.

La deuxième interprétation que nous considérerons est une conséquence directe du texte latin de la Bible. Dans ce texte, le est de la phrase Non est Deus a été compris comme un verbe et a donc généré des réflexions sur l'existence ou la non-existence

5. Cf. Rashi's Commentary on Psalms, édité par Mayer I. Gruber ( « The Brill Reference Library of Judaism», 18), Leyde/Boston, Brill 2004, p. 222-223 ; p. 815816; p. 392-393; p. 829; Weltecke, «Der Narr spricht: Es ist kein Gott», p. 160.

6. Ms. Utrecht UB Cod. 32, 17v, 30v; Utrecht-Psalter, vollständige FaksimileAusgabe im Originalformat der Handschrift 32 aus dem Besitz der Bibliotheek der Rijsuniversiteit te Utrecht, Kommentar, édité par Koert van der Horst/Jacobus H.A. Engelbergt OFM (« Codices Selecti», 75, 75*), Graz, Akademische Druck und Verlagsanstalt 1982; voir Weltecke, «Der Narr spricht: Es ist kein Gott», p. $160-161$. 
d'un dieu. Depuis Augustin d'Hippone, cette phrase a été discutée dans la tradition des commentaires latins. Le débat a connu un premier apogée aux $\mathrm{XII}^{\mathrm{e}}$ et $\mathrm{XIII}^{\mathrm{e}}$ siècles, notamment quand Anselme de Cantorbéry se mit à discuter sérieusement de la question de la non-existence de Dieu et quand Thomas d'Aquin fit le point sur cette argumentation dans sa Somme de théologie. La thèse niant l'existence de Dieu n'a cessé de susciter des réflexions, et ce de manière exponentielle depuis le XII ${ }^{\mathrm{e}}$ siècle ${ }^{7}$.

Prise à la lettre, l'idée de la non-existence de Dieu semblait aberrante à la plupart des savants. L'insensé du psaume se transformait en fou, un fou narcissique, irresponsable et souvent vilain. C'est ainsi qu'est par exemple représenté l'insensé du psaume 14 dans l'initiale d'un manuscrit de La Haye. Dès le $\mathrm{XII}^{\mathrm{e}}$ siècle, ces représentations de l'insensé en fou sont très courantes. Ce fou est quelque peu idiot, il tire la langue, il monte à cheval sur une baguette. Il n'est qu'à moitié vêtu, son derrière est visible. Il ne regarde pas devant lui et va donc probablement chuter ${ }^{8}$. Plus tard, certains attributs comme la marotte et le miroir, ainsi que des habits farfelus et inappropriés sont ajoutés, pour signifier son narcissisme. À la différence du tyran du psaltérion d'Utrecht, ce fou est par définition quelqu'un qui ne croit pas en l'existence de Dieu; il est par définition un athée. Dans la culture du carnaval allemand, il est encore très présent.

À la fin du Moyen Âge, dans sa satire sociale intitulée La nef des fous, Sebastian Brant affirmait que le monde entier était devenu fou - et la non-croyance faisait partie de cette folie: il faut qu'un revenant apparaisse pour convaincre le non-croyant de l'existence de l'enfer. Le texte explique qu'est fou celui qui ne croit pas en la Bible et qui estime au contraire qu'il doit vivre comme s'il n'y avait ni Dieu ni enfer. Le fou méprise tout, doctrine ou sermon, comme s'il était aveugle et sourd'.

7. Pour les sources et la littérature, voir Weltecke, «Der Narr spricht: Es ist kein Gott», p. 432-448.

8. Ps. 52, Ms La Haye, KB, 78 D 38 I, fol. 269 v, c. 1430.

9. « Der ist ein narr der nit der gschrifft/Will glouben die das heil antrifft/und meyned das er leben söll/Als ob kein got wer/noch kein hell/verachtend all predigt und ler/Als ob er nit säh noch hör», Sebastian Brant, Das Narrenschiff. Nach der Erstausgabe (Basel 1494) mit den Zusätzen der Ausgaben von 1495 und 1499 sowie den Holzschnitten der deutschen Originalausgaben, Manfred Lemmer, Tübingen, Niemeyer, 1986, n XI, p. 29-30. 
Dans la Somme des vices et des vertus de Guillaume Peyrault, rédigée au $\mathrm{XIII}^{\mathrm{e}}$ siècle, un chapitre entier est dédié à l'erreur qui consiste à croire qu'il n'y a pas de dieu. Guillaume ne prend pas les adeptes d'une telle erreur au sérieux. Selon lui, cette pensée est stupide et folle. Il ne vaut ni la peine d'argumenter contre cette supposition, ni de punir les incrédules ${ }^{10}$. Quatre cents ans avant Blaise Pascal (décédé en 1662), Guillaume expose l'argumentation suivante: celui qui part du principe qu'il y a un dieu et vit conformément à cette pensée ne peut se nuire, quand bien même il découvrirait après sa mort que ceci était une erreur; par contre, celui qui agit comme s'il n'y avait pas de dieu pourrait être surpris après sa mort, s'il se retrouvait face aux diables de l'enfer.

À l'Université de Paris, on discutait aussi de cette phrase, mais on ne la prenait pas au sérieux. La proposition apparaît dans les Impossibilia de Siger de Brabant (décédé avant 1284). Elle faisait l'objet d'exercice logique pour les étudiants, qui devaient argumenter dans le sens d'une conclusion préétablie, comme pour les autres impossibilia, les autres thèses de cette collection, par exemple que la guerre de Troie continue aujourd'hui encore. Il est évident que l'assertion est erronée ${ }^{11}$. Les savants scolastiques sont d'accord sur ce point: seul le fou dit qu'il n'y a pas de dieu. C'est pourquoi d'ailleurs cette thèse n'a jamais été censurée, contrairement à bien d'autres.

Depuis cette époque, la perception de l'athéisme a radicalement changé; l'attribution de la rationalité et de l'irrationalité a été inversée, comme en témoignent les arguments des nouveaux athées par exemple. Le tyran, l'imbécile ou le fou est, selon eux, celui qui croit en Dieu. Il nous semble que l'historien devrait se contenter de constater ces faits, sans pour autant en juger. Du moment où les conceptions et les points de vue changent, les chercheurs qui s'entêtent à prendre leurs convictions pour des vérités absolues encourent le risque de faire fausse route.

10. Guillelmus Peraldus, Summa virtutum ac vitiorum, éd. Rodolphus Clutius, t. I-II, Paris, Petit, 1629, p. 46.

11. Siger de Brabant, «Impossibilia», dans, Id., Écrits de logique, de morale et de physique, éd. Bernardo Bazán, Louvain/Paris, Publications universitaires/ Beátrice-Nauwelaerts («Philosophes médiévaux», 14), 1974, p. 67-97. 


\section{Doutes}

Très souvent, on allègue que le doute en général éveillait les soupçons des autorités médiévales. Il convient cependant de faire la part entre les différents types de doutes. D'abord, les termes médiévaux qui signifient le doute renvoient à une attitude indécise entre deux positions. Ils ne signifient pas nécessairement l'absence de conviction. Perturbations dans des relations entre seigneur et serf, entre amis ou amoureux, dissensions et manque de fiabilité faisaient partie du doute autant que la peur, l'hésitation ou la versatilité. D'autre part, certains doutes ont été interprétés comme une hésitation rationnelle et évalués comme une compétence sociale. Par ailleurs, la scolastique médiévale a connu une forme de doute méthodique qui a ensuite été apparenté aux concepts modernes de doute méthodique et considéré comme un moteur de la recherche.

Comment interpréter la très célèbre norme juridique contenue dans les Décrétales du Pape Grégoire IX datant de 1234, qui affirme: «Dubius in fide infidelis est, nec eis omnino credendum est, qui fidem veritatis ignorant $»^{12}$. Cette phrase signifie-t-elle «Celui qui doute de la foi est un non-croyant» ? En outre, le doute est-il condamné et puni dès l'époque de Grégoire IX? On rencontre cette supposition dans la littérature scientifique. Pourtant, cela paraît plutôt invraisemblable. La recherche en droit ecclésiastique a montré qu'il faut interpréter cette phrase dans un contexte différent. La proposition «Dubius in fide infidelis est » provient de fausses Décrétales ou Pseudo-Isidoriana rédigées au Haut Moyen Âge, qui furent intégrées plus tard au droit canon. Dans les Pseudo-Isidoriana, il était question de manque de crédibilité devant la justice, car infidelis signifiait infidèle ${ }^{13}$.

12. Decretal. Gregor, IX, Lib., V, Tit. VII, De haereticis, Cap. I, dans Emil Richter et Emil Friedberg (dir.), Corpus Iuris Canonici, II, Decretalium Collectiones, Leipzig, Tauchnitz, 1879-1881 (Réimpr. Graz, Akademische Drucku. Verlagsanstalt 1955).

13. Epistola Primi Sixti, dans Paul Hinschius (dir.), Decretales PseudoIsidorianae et Capitula Angilramni, Leipzig, Tauchnitz, 1863 (Réimpr., Aalen, Scientia, 1963), p. 107: «De cetero placuit propter malorum hominum infestationes, ut in accusatione domini ministrorum primo persona, fides, vita et conversatio blasphemantium enucleatim perscrutetur. Nam fides omnes actus hominis precedere debet, quia dubius in fide infidelis est, nec eis omnino esse credendum qui veritatis fidem ignorant, nec recte conversationis vitam ducunt: quoniam tales facile indifferenter lacerant et criminantur recte et pie viventes, ideo suspicio eorum discutienda est primum et corrigenda.» 
Or, c'est bien le même sens qui est mobilisé dans le nouveau contexte des Décrétales de Grégoire IX, c'est-à-dire celui de la poursuite des hérétiques au XIII ${ }^{\mathrm{e}}$ siècle. L'hérésie était l'adhésion délibérée et obstinée à une doctrine ou à une manière de vivre qui étaient formellement condamnées par l'Église. Toutes les déviances n'étaient pas interprétées comme hérésie. Quoi qu'il en soit, l'hérésie était par définition un délit criminel, tant pour le droit canon que pour le droit séculier.

Ainsi, «Dubius in fide» doit vraisemblablement s'interpréter dans le contexte de la mobilisation par le pape Grégoire des tribunaux de l'Inquisition contre les mouvements hérétiques de son temps. Le pape voulait priver les mouvements hérétiques notamment les Cathares ou les Vaudois - de leurs renforts. Celui qui se montrait indécis par rapport à cette politique du pape, c'està-dire «infidèle», infidelis, était considéré comme un hérétique, de même que ses sympathisants et ses soutiens. La notion juridique d'hérésie avait donc été élargie.

L'étude des manuels de l'Inquisition, qui ont constitué la base juridique de la persécution et qui décrivaient les typologies de personnes recherchées par les inquisiteurs, aboutit à des résultats sans équivoque. Les personnes qui se contentaient d'exprimer des doutes religieux de manière générale n'étaient pas persécutées; elles n'étaient pas réputées hérétiques. Les manuels et les procèsverbaux d'audition révèlent que les Inquisiteurs ne recherchaient pas des athées. De même, la phrase «il n'y a pas de Dieu» n'est pas mentionnée dans les manuels de l'Inquisition. Les doutes ou la non-croyance n'étaient pas un crime ${ }^{14}$. Il en allait de même du blasphème, qui était dénoncé comme méfait criminel au XIII ${ }^{\mathrm{e}}$ siècle. Le blasphème n'était pas poursuivi par l'Église mais par des tribunaux séculiers, et il fut puni de plus en plus sévèrement à partir de l'époque moderne ${ }^{15}$. Mais la simple phrase «il n’y a pas de dieu » n'était pas considérée comme un blasphème. Blasphémer était un acte langagier agressif, une malédiction qui défiait Dieu, par exemple au cours de jeux de hasard dans les tavernes. Les recherches de Schwerhoff, Casagrande/Veccio et d'autres l'ont

14. Voir Weltecke, «Der Narr spricht: Es ist kein Gott», p. 330-362.

15. Corinne Leveleux-Teixeira, La parole interdite: le blasphème dans la France médiévale (XIII -XVI siècle), Paris, De Boccard, 1995. 
démontré ${ }^{16}$. Un blasphème très répandu, celui qui proclamait «je nie Dieu», signifiait désavouer sa relation à Dieu et non pas nier son existence.

\section{Concepts de doute et de Reniement de Dieu}

Avant de développer le sujet du doute et du reniement de Dieu, on acceptera donc le principe selon lequel l'athéisme n'était un problème ni pour les savants ni pour les Inquisiteurs, bien que les doctrines hérétiques fussent persécutées. Ceci peut sembler paradoxal, mais il faut reconnaître que les valeurs religieuses n'étaient pas organisées au Moyen Âge comme à l'époque moderne. Au Moyen Âge, la non-croyance apparaît comme un problème social et religieux dans la littérature, particulièrement dans la littérature spirituelle. Dans ce qui suit, nous esquisserons ces problématiques. Le discours médiéval a traité des émotions en les ordonnant sous différents concepts.

\section{«Acedia»}

L'acédie, considérée comme un péché capital, a été étudiée depuis longtemps, particulièrement depuis l'enquête de Klibansky, Panofsky et Saxl sur la mélancolie. Certains érudits ont démarqué l'acédie médiévale de la mélancolie sublime de l'époque moderne et de l'acédie spirituelle du monachisme ancien. Comparée à ces deux autres conceptions de l'acédie, l'acédie de la littérature spirituelle du Haut Moyen Âge et du Moyen Âge tardif semble signifier une simple paresse. L'acedia n'est cependant pas une paresse commune. Elle a un effet sur l'attitude des personnes envers la foi et l'Église. Avec l'acedia, on décrivait des émotions telles que la répugnance ou l'indifférence à l'égard de Dieu. «He loveth not his god ne dredith not his god», expliquait au sujet de l'acédie un traité anglais sur les

16. Carla Casagrande, Silvana Vecchio, Les péchés de la langue: discipline et éthique de la parole dans la culture médiévale, Paris, Éd. du Cerf, 1991; Carla Casagrande, Silvana Vecchio, Histoire des péchés capitaux au Moyen Âge, Paris, Aubier, 2003 (première édition 2000). Gerd Schwerhoff, Zungen wie Schwerter. Blasphemie in alteuropäischen Gesellschaften 1200-1650, Constance, UVKVerlags-Gesellschaft, 2005. Cf. Weltecke, "Der Narr spricht: Es ist kein Gott», p. 315-316. 
péchés, rédigé au $\mathrm{XIV}^{\mathrm{e}}$ siècle ${ }^{17}$. Selon les manuels de confession, il fallait demander au pénitent s'il répugnait à aller à l'église et à prier (taedium, displicentia), s'il aimait écouter le sermon ou s'il s'ennuyait. Les auteurs de la littérature édifiante envisageaient les émotions et les sentiments contraires au christianisme comme une chose naturelle.

L'acédie ne devenait un péché capital que lorsque quelqu'un approuvait consciemment ces attitudes que sont la répugnance, l'ennui, le déplaisir et même l'indifférence totale envers le culte. Or, même envisagée comme un péché capital, l'acédie n'était pas soumise à l'Inquisition. Cette différence de traitement est très importante. En effet, les inquisiteurs interrogeaient les suspects qui méprisaient les sacrements. Certaines personnes en dénonçaient d'autres, parce qu'elles ne regardaient pas les sacrements pendant la messe. Ces personnes n'étaient cependant pas interrogées parce qu'elles se désintéressaient ou doutaient des sacrements, mais, parce qu'on pouvait les soupçonner d'une hérésie, par exemple de l'hérésie des Vaudois, des Hussites ou des Lollards.

L'acédie, quant à elle, n'était pas une hérésie. Ceux qui s'y livraient abandonnaient consciemment tout intérêt pour la communauté et la foi. Dieu et la culture religieuse les rebutaient par définition. Le clergé ne pouvait qu'exhorter ou réprimander. Ce n'est qu'à l'époque moderne qu'on se mit à poursuivre les gens pour les faire aller à l'église. Au Moyen Âge, la faculté de contrôle était limitée. On laissait les gens à leur vie, même pendant quarante ans et plus, comme on le racontait ${ }^{18}$.

\section{«Tentation» et «blasphème de cour»}

Ces deux notions servaient à décrire des idées qui hantaient un homme à l'improviste et sur lesquelles il n'avait pas de contrôle ${ }^{19}$. Elles datent de la littérature monacale primitive, dans laquelle elles signifient de telles émotions indésirables. Au tout début,

17. Richard O. Carm Lavynham, A Litil Tretys on the Seven Deadly Sins, éd. Johannes van Zutphen, Rome, Institutum Carmelitianum, 1956, p. 17.

18. Pour la littérature et les sources, voir Weltecke, «Der Narr spricht: Es ist kein Gott», p. 369-378. Adelheid Langmann, Die Offenbarungen der Adelheid Langmann, Klosterfrau zu Engelthal, éd. Philipp Strauch, Strasbourg-Londres, Trübner 1878, p. 44-46.

19. Voir Weltecke, «Der Narr spricht: Es ist kein Gott», p. 378-411. 
ces émotions furent qualifiées de tentations (temptationes ou impugnationes). «Tentation» et «blasphème de cœur» imprègnent la culture monacale aussi bien occidentale qu'orientale et, dans un premier temps, les deux termes demeurèrent confinés à cette culture. Ce n'est qu'à partir du Moyen Âge que des écrivains ont aussi accordé aux laïcs la possibilité de vivre les tentations comme des expériences spirituelles. Le mot «tentation» n'est pas précis. Il comportait toutes sortes d'émotions, mais il englobait aussi explicitement des doutes religieux radicaux.

Dans la tradition monacale, le bénédictin Otloh de Ratisbonne a livré un récit célèbre de ses propres tentations. Dans ses écrits, il met ironiquement en scène Dieu signifiant à Otloh qu'il est un sceptique zélé (le syntagme amator dubitationis faisant jeu de mots avec l'ami de la sagesse, le philosophe $)^{20}$. Dans son Liber de Temptatione rédigé autour de 1070, Otloh expose ses propres doutes quant à la véracité des Écritures saintes et à l'existence de Dieu. Il lui semble que ces doutes lui sont chuchotés par un démon au creux de l'oreille, annihilant sa propre volonté. La souffrance qu'il ressent et l'état délétère du monde sont la preuve qu'il n'y a aucun guide tout-puissant. Henri Suso, du Lac de Constance (1297-1366), fournit un second exemple. Dans son autobiographie, il rapporte avoir eu de «mauvaises idées» sur la foi pendant neuf années ${ }^{21}$. Otloh et Henri affirmaient raconter leurs expériences pour consoler ceux qui enduraient les mêmes souffrances. Comme témoins de cette attitude, on peut encore mentionner quelques autobiographies, également de la culture monacale moyen-orientale, qui se proposaient le même objectif de consolation d'autrui. Il faut cependant constater que de

20. Otloh von St. Emmeram, Liber de temptatione cuiusdam monachi. Untersuchung, kritische Edition und Übersetzung, éd. Sabine Gäbe, Berne, Peter Lang («Lateinische Sprache und Literatur des Mittelalters», 29), 1999, p. 286.

21. Heinrich Seuse, Deutsche Schriften, Stuttgart, éd. Karl Bihlmeyer, Kohlhammer, 1907, p. 61-62: «Under andren sinen liden waren drú inrú liden, dú im do vil pinlich waren. Dero was eins unreht invelle von dem globen. Im viel in sinen gedank also: wie moht got mensch werden? und des gelich menges. So er ieme begegnete, so er ieme verierrete. In dieser anvehtung liess in got wol uf IX jar mit schriendem herzen und weinenden ogen uf zuo gote und ze allen heiligen umb hilf. Ze jungst neiswen, do es got zit duhte, do half er im genzlich dur von, und war im got groessú vestekeit und erlúhtunge des globen. Daz ander inrlich liden war ungeordnetú trurkeit. Im waz emzklich als swer in sinem gemuete, als ob ein berg uf sinem herzen leg; und waz daz ein teil da von: sin geswinder abker waz so scharpf, daz siner leblichen natur vil gross gedrang dur von beschah. Disú not werete im wol VIII jar [H.v.m.]». 
tels témoignages sont rares. Il était par contre un peu plus facile de parler de ces «tentations» dans les histoires édifiantes ou exempla. $\mathrm{Au}$ début du XIII ${ }^{\mathrm{e}}$ siècle, le Cistercien Césaire de Heisterbach a réalisé une collection d'exempla pour les novices et les moines. L'intégralité de la quatrième partie de son Dialogus Miraculorum a trait aux tentations, aux temptationes. Selon Césaire, beaucoup de gens se brisaient contre elles et tombaient dans la desperatio, le désespoir.

Dans l'un de ces exempla, Césaire raconte comment une jeune recluse commença à faillir dans sa foi («in fide nutare»). Belle et riche jeune fille, cette recluse s'était imposé une vie religieuse, allant à l'encontre de l'opinion de ses parents qui voulaient la marier. Cependant, peu après avoir réussi à se faire enfermer, elle éprouva une crise spirituelle. Elle ressentit d'abord une forte aversion envers la prière, une répulsion à l'encontre de la vie de recluse: elle était en proie à l'acédie. À l'abbé qui l'avait prise en charge et lui avait demandé comment elle se portait, elle avait répondu qu'elle se portait mal et qu'elle ne savait plus pourquoi elle avait choisi la réclusion. "Sûrement pour Dieu», lui répondit l'abbé. "Qui sait, s'il y a un dieu, des anges ou un ciel», objecta-t-elle. «Personne ne l'a vu, personne n'est revenu du royaume des cieux pour le confirmer». Exhortée par l'abbé, elle continua en alléguant: «je dis ce que je pense et je ne crois que ce que je vois». Elle dit de plus que la vie religieuse lui était devenue insupportable ${ }^{22}$. Comme très souvent dans de telles histoires, la recluse eut une vision. Elle quittait son corps, elle se voyait elle-même et apercevait en même temps tout ce dont elle avait douté durant son existence: les anges, le ciel et Dieu.

Dans cette histoire, ainsi que dans les écrits d'Otloh, les propositions sont claires et sans équivoque; elles parlent de doutes

22. Cesarius von Heisterbach, Dialogus Miraculorum, éd. Joseph Strange, t. I-II, Köln, Heberle, 1851 (Réimpr. Ridgewood, New Jersey, Gregg Press, 1966), distinctio IV, ch. xxxix: «Male vivo, male valeo, et quare vel propter quem hic reclusa sim, prorsus ignoro. Cui cum Abbas diceret: Propter Deum et propter regnum coelorum; illa respondit: Quis scit, si Deus sit, si sint cum illo angeli, animae, vel regnum coelorum? Quis ista vidit? quis inde rediens visa nobis manifestavit? Huiuscemodi sermones ut Abbas audivit, totus contremuit, conversusque ad virginem ait: Quod est quod loqueris, soror? Signa cor tuum. Respondit illa: Ego loquor sicut mihi videtur. Nisi videam ista, non credam. Rogo ut sinas me exire, quia reclusionem hanc diutius sustinere non valeo.» 
quant à l'existence de Dieu et des anges, comme l'explique le titre ( «reclusa Deum et angelos esse dubitans »). Cette histoire est insérée dans un récit-cadre, un entretien entre un novice et un moine. Dans le Dialogus Miraculorum, le novice qui parle avec son maître de ce cas s'épouvante en pensant que Dieu puisse permettre qu'une âme chaste soit tentée à ce point.

En outre, des sources nous apprennent que certains laïcs n'étaient pas non plus convaincus de l'existence de Dieu. Peter de Cornwall, le prieur de Holy Trinity à Aldgate, a rédigé son Liber revelationum autour de l'an 1200. Il justifiait son ouvrage par l'intention d'aider les gens en proie à des doutes quant à l'existence de Dieu. Il expliquait que la plupart des hommes ne croient plus aux idoles et savent qu'il n'y a qu'un Dieu. Il ajoutait cependant que beaucoup de gens pensent qu'il n'y a pas de Dieu, que le monde est éternel et que le cours du monde est déterminé par le hasard et non par la providence de Dieu. Selon lui, beaucoup d'hommes croient qu'il n'y a ni anges ni démons, ni rien de spirituel et d'invisible. Peter considérait ces idées comme une ignorance infantile des origines de la vie humaine. Il expliquait avoir compilé les visions qui composent son ouvrage pour instruire les gens simples ${ }^{23}$.

Encore une fois, nous constatons que la vision est l'antidote à la tentation; elle est pourtant incontrôlable. Un autre moyen recommandé pour combattre les doutes est de prendre ses distances par rapport aux idées incontrôlables. Les livres spirituels de consolation ou les directeurs de conscience expliquaient que les doutes ou la non-croyance ne valent pas la peine qu'on en parle. Il ne vaut pas non plus la peine d'y réfléchir, que ce soit spirituellement ou rationnellement. Il fallait au contraire les repousser sans même en parler. De nombreuses descriptions dramatiques dépeignent toutefois la détresse des gens incertains quant à leur foi. Les confessés souffraient, et les confesseurs les appelaient au calme.

Cependant, cette inquiétude avait été provoquée par une pression religieuse croissante. À cet égard, on a déjà mentionné l'Inquisition et la criminalisation du blasphème. Mais à y réfléchir plus attentivement, il semble que cette énorme angoisse de pécher soit une conséquence directe de la confession elle-même. Selon

23. Peter von Cornwall, Liber revelationum. Lambeth Palace Library, ms. 51, f. $2 \mathrm{r}^{\circ}$ 
le concile du Latran IV, il devait être demandé au confessé s'il croyait «fermement» et s'il ne doutait pas. Ce sont les directeurs de conscience eux-mêmes qui ont fait naître le problème, et ils en étaient conscients. Ils faisaient tout ce qui était en leur pouvoir pour justifier l'idée de doute ou de non-croyance. Par ailleurs, ils différenciaient de telles idées de l'hérésie et du blasphème criminel, et ils les traitaient avec clémence. Ils demandaient aux prêtres de ne pas être trop sévères avec leurs confessés. De plus, ils critiquaient les confesseurs qui écartaient les confessés de la foi en les réprimandant ou, pire, en les poussant au désespoir théologique, à la desperatio.

Le théologien et chancelier de l'université de Paris Jean Gerson s'occupa de cette question. Il parla lui aussi de certaines idées blasphématoires qui échappaient à tout contrôle : il est impossible de les contrôler, tout comme on ne peut capturer le vent. Gerson narre l'exemplum d'un ermite qui était tourmenté par des idées horribles et blasphématoires. De fait, les ermites sont les protagonistes idéaux de tels exempla, au même titre qu'une religieuse ou une recluse, parce qu'il n'y a pas de doute sur leur vertu et leur bonne intention de croire ${ }^{24}$.

Pourtant, ni les ermites, ni les religieuses, ni les recluses n'appartiennent au monde érudit des lettrés. L'étude de leurs cas permet de fait une approche du problème du doute religieux. Pourtant Gerson ne rapporte pas exactement ce que l'ermite pense. Celui-ci n'ose ni le dire ni l'écrire. On apprend seulement que ce doute le plonge dans l'angoisse du péché et presque dans la desperatio. L'ermite a eu de la chance: il n'est pas tombé sur un confesseur qui damnait les confessés au lieu de les consoler. Le prêtre averti a d'abord examiné si l'ermite adhérait à ses idées en toute conscience. Si tel n'était pas le cas, il les expliquait comme des tentations du diable. Au moyen d'une déclaration à haute voix, l'ermite devait se distancer de son idée: «Ceci n'est pas ce que je pense !» Ensuite il se sentirait soulagé.

$\mathrm{Au} \mathrm{XV}^{\mathrm{e}}$ siècle, le prédicateur de Strasbourg, Jean Geiler de Kaysersberg, a précisé comment il convient d'interpréter la notion

24. Jean Gerson, Contre les tentations de Blasphème, dans Id., Euvres Complètes, éd. Palémon Glorieux, t. vII, vol. 1, Paris, Desclée, 1973, n 336 , p. $412-416$. 
de «blasphème de cœur», terme qu'il utilisait pour qualifier la tentation des laïcs. Celui qui voit le sacrement peut se demander: «comment est-il possible que Dieu soit là-dedans?» Quand il contemple le crucifix, il peut penser: "comment est-il possible que Dieu puisse mourir - cela est vraiment trop peu digne d'un dieu !» En voyant la Vierge, il peut se demander : «comment est-il possible qu'elle soit restée vierge?» Bref, il peut penser que tout ce que la foi enseigne n'est qu' «escamotage $»^{25}$. Contre toute attente, Jean Geiler affirme cependant que ces doutes ne sont pas graves, même s'ils semblent être radicaux. Il reprend le propos de Jean Gerson, affirmant que «l'homme ne peut pas contrôler ses idées $»^{26}$. De telles idées deviennent un péché - et non un crime - quand des gens les affirment consciemment et rationnellement. Stephan de Landskron, frère augustin viennois du $\mathrm{XV}^{\mathrm{e}}$ siècle, conseillait de ne pas confesser de telles idées. En particulier les religieuses, les moines ou les béguines ne devaient pas en parler à un simple prêtre pour ne pas provoquer son irritation ${ }^{27}$. Il n'est de fait pas étonnant qu'il soit si difficile de retrouver des témoins de ces problématiques dans les sources conservées aujourd'hui, puisqu'on évitait d'en parler.

Certaines personnes étaient en proie aux doutes ou à la noncroyance. Ces doutes avaient trait aux fondements mêmes de la religion et ils étaient peut-être immédiatement provoqués par la

25. Johannes Geiler von Kaysersberg, Das buch der sünden des munds. Von dem hochgelerten Doctor Keisersperg, die er nennt Die blattren am mund, davon er .xxix. predigen und leeren gethon hat. Auch darby Doctor Keiserspergs Alphabet in .xxiii. predigen geordiniert, Straßburg, Grieniger, 1518, $\mathrm{n}^{\circ}$ XIX.

26. Jean Gerson, Traité des diverses tentations de l'ennemi, dans Id., Euvres Complètes, éd. Palémon Glorieux, t. vII, vol. 1, Paris, Desclée, 1973, n 324, p. 343-360, ici p. 353. «Aucunefoys l'ennemi trouble la pensee par y enuoyer grant nombre de pensees diuerses, laydes et abhominables, adfin que la personne soit en doubte et paour d'estre continuellement en pechié; et luy dit l'ennemi que c'est trop grant pechié quant en disant ses heures ou en seruant Dieu en espicial tesses pensees se viennent; et enhorte que la personne les veulle du tout oster et que rien ne face fors labourer a ce; car ils scet bien que non plus que non pourrait arrester le vent en sa main, en plus ne peut on toudis oster ses pensees qu'elles ne viennent; mais au consentir est la pechié, c'est a dire quant la personne veult bien auoir tesses pensees; mais tant qu'elles luy desplaisent, il n'i a point peril de pechié morteil, ainsoiz y a grant merite.»

27. Stephan von Landskron, Die Hymelstrasz. Mit einer Einleitung und vergleichenden Betrachtungen zum Sprachgebrauch in den Frühdrucken (Augsburg 1484, 1501 und 1510), éd. Gerardus J. Jaspers, Amsterdam, Rodopi («Quellen und Forschungen zur Erbauungsliteratur des späten Mittelalters und der frühen Neuzeit», 13), 1979, f. 22 r. 
confession et l'Inquisition. On ne trouvait cependant personne qui voulût les mettre par écrit ou même les raconter. Césaire de Heisterbach avait décidé que de tels récits étaient plus nuisibles que profitables. C'est pourquoi il se contentait de narrer quelques exemples, plutôt moins qu'il n'en savait, selon son propre aveu. Il n'était fait mention de ces idées que lors d'entretiens entre novice et maître, entre laïc confessé et prêtre confesseur, entre moines ou même entre laïcs, mais toujours à voix basse. Jamais ces idées n'étaient discutées sur le plan théologique. Elles n'étaient pas considérées comme criminelles, mais elles n'étaient pas non plus prises au sérieux.

\section{«Murmur» et «impatientia»: Protestation et théodicée}

Il existait cependant un autre concept de doute et de noncroyance qui, à la différence de la tentation, n'était pas tu et, de ce fait, était considéré comme un péché28. Il était né de la protestation contre l'injustice et mettait en doute l'omnipotence de Dieu, au vu de la souffrance humaine. Au Moyen Âge, on apportait trois types de réponses à la question qui demandait comment le malheur pouvait échoir à un innocent ou le bonheur à un malfaiteur, et comment les calamités pouvaient être expliquées. En premier lieu, on supposait que le malheur était la punition d'un péché commis, et ceci suffisait à mettre un terme à la contradiction entre la bonté de Dieu et l'expérience de la souffrance. Deuxièmement, on pouvait élucider cette aporie au moyen de la solution de Boèce (environ 475/80 - jusqu'à 525). Celui-ci, incarcéré pour cause de trahison, attendait la peine capitale. Dans cette situation, il s'était demandé comment, s'il y a un Dieu bon et tout-puissant, le mal pouvait sembler prévaloir $^{29}$. Dame philosophie lui avait répondu qu'en réalité tout est bien à sa place. Les hommes bons réussissent et ont du pouvoir, les malfaiteurs n'obtiennent rien. Le mauvais tyran peut certes agir à sa guise, mais il ne peut faire et obtenir ce qu'il veut en réalité, car l'objet du désir naturel et la finalité de l'homme

28. Voir Weltecke, «Der Narr spricht: Es ist kein Gott», p. 412-432.

29. Boèce, De consolatione philosophiae. Opuscula theologica, éd. Claudio Moreschini, München/Leipzig, Saur («Bibliotheca Teubneriana»), 2000, p. 100: «Sed ea ipsa est vel maxima nostri causa maeroris quod, cum rerum bonus rector exsistat, vel esse omnino mala possint vel impunita praetereant...». 
sont le bien suprême. Or le tyran ne peut jamais accéder à un tel bien. Ici, Boèce ne discute pas de la bonté de Dieu ou de sa toutepuissance, mais il se demande au moyen de quels critères les gens évaluaient le pouvoir ou le succès. La troisième possibilité pour justifier le malheur et les calamités qui s'abattent sur l'homme était enfin de les attribuer aux astres. L'astrologie avait une importance croissante depuis le Haut Moyen Âge, de plus en plus de gens y avaient recours.

L'existence même de ces théories montre que la contradiction entre la bonté de Dieu et l'état délétère du monde avait été très clairement perçue. Cependant, seul un petit nombre de savants traitait la question de la cause du mal. Thomas d'Aquin avait envisagé les malheurs du monde comme un possible argument contre l'existence de Dieu ${ }^{30}$. On peut également penser aux lettres de Pétrarque relatives à la peste, à l'affliction que lui cause la mort d'autant de gens innocents et bons ${ }^{31}$. On peut aussi se tourner vers les lettres de lamentation du frère prêcheur Riccoldo da Monte di Croce (environ 1243-1320), le dernier État latin d'Orient après la conquête de Saint-Jean-d'Acre en $1291^{32}$. Bien que les textes de Pétrarque et de Riccoldo soient très intéressants comme témoignages autobiographiques, ils restent cependant très marginaux, tout comme le sont les autobiographies des moines qui doutent. Dans ce cas également, la littérature spirituelle est plus prolifique.

Les auteurs de la littérature spirituelle interprétaient de telles querelles et de tels doutes comme des «râles» (murmur) ou des

30. Thomas d'Aquin, Summa Theologiae, t. I-I, qu. 2, art. III: «Ad tertium sic proceditur. I. Videtur quod Deus non sit. Quia si unum contrariorum fuerit infinitum totaliter destruetur aliud. Sed hoc intelligitur in hoc nomine Deus, scilicet quod sit quoddam bonum infinitum. Si ergo Deus esset, nullum malum inveniretur. Invenitur autem malum in mundo. Ergo Deus non est. 1. Praeterea, quod potest compleri per pauciora principia, non fit per plura. Sed videtur quod omnia quae apparent in mundo, possunt compleri per alia principia, supposito quod Deus non sit; quia ea quae sunt naturalia, reducuntur in principium quod est natura; ea vero quae sunt a proposito, reducuntur in principium quod est ratio humana, vel voluntas. Nulla igitur necessitas est ponere Deum esse.»

31. Pétrarque, Francisci Petrarcae Epistolae De Rebus Familiaribus et Variae, éd. Giuseppe Fracasetti, t. I-III, Florence, Le Monnier, 1859, t. I, p. 440-441.

32. Riccoldo da Monte di Croce, «Lettres de Ricoldo de Monte-Croce. Ricoldi de Monte Crucis Ordinis praedicatorum Epistolae V commentatoriae de perditione Acconis 1291 », éd. Reinhold Röhricht, Archives de l'Orient latin, 2 B, 1884, p. $258-296$. 
marques d' «impatience» (impatientia). À leur grand regret, ils leur semblaient très répandus. Ils les regardaient comme un vice ou un péché contre le premier commandement. Selon Jean Gerson dans son Opus tripartitum, au premier commandement contreviennent tous ceux qui grognent contre la volonté de Dieu et qui croient qu'il n'est pas tout à fait juste et bon. D'autres lettrés d'Europe occidentale approuvaient cependant ces grognements. Dans les instructions pour bien mourir, l'Ars Moriendi, on voit un malade tourmenté par l'impatience. Il donne des coups de pied autour de lui. Il ne veut de la pitié de personne, pas plus que des paroles hypocrites de sa visiteuse qui s'écrie: «Quelle punition il subit!», (ecce quantum penam patitur). Le mourant n'est pas d'accord avec elle, il n'accepte pas l'idée qu'une telle souffrance puisse être une punition, en tout cas une punition juste. Un petit démon s'en réjouit, en observant son œuvre par la droite (il persifle entre ses dents): «Comme je l'ai bien trompé» (Quam bene decepi eum) ${ }^{33}$. Dans les explications de Johann von Staupitz, le père confesseur de Martin Luther, on lit que selon les théologiens, l'impatientia éprouvée dans une maladie mortelle peut donner lieu à une perte totale de la foi. Johann von Staupitz dit expressément que la souffrance peut faire naître dans le cœur du malade l'idée «qu'il n'y a pas de Dieu, qu'il n'y a pas de justice $»^{34}$.

Râler ainsi était un péché. Celui qui râle a la réputation d'être fou, comme l'affirmait déjà la Somme de Guillaume Peyrault mentionnée auparavant. Les exempla qui racontent les protestations contre l'injustice de Dieu et du monde sont très souvent empreints d'une certaine drôlerie. Frantiček Graus et d'autres les ont rassemblés. Dans la Tabula Exemplorum, il y a par exemple, une petite histoire qui parle d'aumônes: un comédien malade laisse à des riches et des puissants une fortune considérable, composée de chevaux et d'autres biens. En réponse à la question demandant pourquoi il ne les donne pas à des pauvres, il réplique qu'il agit selon le conseil des prêtres qui prêchent qu'il faut imiter Dieu. Comme Dieu lui-

33. Ars Moriendi, Munich, Bayerische Staatsbibliothek, xylogr. 16, f. $2 \mathrm{v}^{\circ}$, f. $6 \mathrm{v}^{\circ}$, ill. 3,5 .

34. Johann von Staupitz, Johann von Staupitzens sämmtliche Werke, I. Deutsche Schriften - De imitanda morte Jesu Christi libellus (Ein buchlein von der nachfolgung des willigen sterbens Christi) [1515], éd. Joachim K. F. Knaake, Potsdam, Krausnick, 1867, p. 64. 
même accorde les biens de ce monde aux riches, lui, le comédien, fera de même ${ }^{35}$.

Cette histoire prête à rire, car les comédiens ne sont pas des gens sérieux. La plaisanterie se fait aux dépens du prêtre, dans un sermon proféré par le prêtre lui-même. Une histoire de la collection du Franciscain Johannes Pauli, qui a vécu au Xve siècle, est construite de la même manière. Un prêtre dit à un malade, pour le consoler, que l'amitié de Dieu se montre dans la souffrance qu'il offre aux hommes. Le malade répond, nonchalant, que dans ce cas il n'est pas surpris que Dieu ait aussi peu d'amis ${ }^{36}$. Les deux histoires révèlent que les gens n'étaient pas convaincus par les tentatives d'apaisement des pasteurs - et que les réformateurs le savaient bien. De tels exempla étaient destinés à faire rire le public, mais ils devaient aussi faire comprendre que cette attitude de doute et les protestations contre Dieu sont pure folie.

Dans d'autres Églises médiévales, on testait des mesures différentes. Les premiers théologiens de l'Église syrienne orthodoxe discutaient sérieusement de la raison du massacre de tant d'innocents en l'an 1141 à Édesse. On se demandait si Dieu avait laissé faire et pourquoi. Ici aussi, les gens murmuraient parce qu'ils ne comprenaient pas pourquoi les religieux et les enfants - symboles de l'innocence - avaient été assassinés. On murmurait que la toutepuissance de Dieu ne s'était pas étendue jusqu'à eux. Dans le monde latin, on ne souhaitait pas aborder de tels sujets, ni théologiquement ni philosophiquement, à quelques rares exceptions près. Ainsi, ces idées ne nous sont parvenues que comme un murmure ou un bruit de fond.

\section{Conclusion}

Les doutes religieux et la non-croyance n'ont pas été poursuivis par l'Inquisition. Pourtant, il est incontestable que de nombreuses

35. La Tabula exemplorum secundum ordinem alphabeti. Recueil d'exempla compilé en France à la fin du XIII e siècle, éd. Jean T. Welter, Paris/Toulouse, Occitania, 1927 (Réimpr. Genève, Slatkine, 1973), n 76 (Elemosina): «Vos sacerdotes predicatis nobis quod debemus esse imitatores Dei; Deus autem omnia bona mundi divitibus largitur non pauperibus et ego similiter facio de rebus meis ».

36. Johannes Pauli, Schimpf und Ernst, éd. Johannes Bolte, t. I-II, Berlin, Stubenrauch, 1924 (Réimpr. Hildesheim, Olms, 1972), t. II, p. 53. 
personnes ont été persécutées en tant qu'hérétiques à la fin du Moyen Âge, parce qu'elles n'étaient pas convaincues par les doctrines théologiques de l'Église. Les hérétiques étaient cependant accusés pour avoir défendu des doctrines alternatives erronées. De nombreuses sources révèlent de toute évidence que la poursuite du blasphème n'était pas une chasse aux athées durant le Moyen Âge, même si elle l'est devenue plus tard. Les critiques radicales de l’Église et de certaines doctrines théologiques ne sont bien sûr pas absentes de l'horizon médiéval, mais elles n'apparaissent que dans des contextes alternatifs hérétiques. Tel n'est pas le thème qui est traité ici. En tant que telle, la non-croyance n'était qu'un vacuum, un vide.

Doutes religieux et non-croyance ont été traités par la littérature, particulièrement par la littérature spirituelle. Des personnes tourmentées par des idées et des doutes étaient consolées; lorsqu'elles n'éprouvaient aucun scrupule, elles pouvaient être exhortées ou même réprimandées. Les savants étaient familiers de ces idées et de ces émotions. Mais ni philosophes ni théologiens ne les prenaient au sérieux. En effet, les clercs ont fait obstacle à toute discussion rationnelle de ces questions durant des siècles, jusqu'à l'époque moderne.

À l'époque moderne, les lettrés assemblèrent un certain nombre d'arguments traditionnels; le discours hérétique, le discours critique et le discours athée confluèrent pour créer une mode intellectuelle. Dans ce contexte, les savants se situaient à l'avant-garde de la société et prétendaient qu'il en avait toujours été ainsi : en tant que sapientes, ils prétendaient avoir toujours déchiffré les impostures du clergé. Les pages précédentes ont permis de présenter un tableau très contrasté. La vérité et la réalité changent constamment; les érudits croient seulement les comprendre et être toujours en mesure de les dominer.

dorothea.weltecke@uni-konstanz.de 